\title{
A Study on Visual Outcome After Surgery for Traumatic Cataract
}

\author{
Dr. Jyoti Bhuyan ${ }^{1}$, Dr.Baby Deka², Dr. Ashvini Vishnuji Kadhao ${ }^{3}$, \\ Professor,Regional Institute Of Ophthalmology, Gauhati Medical College.
}

\begin{abstract}
Background: Ocular trauma is an important, preventable public health problem worldwide. It is the single most important cause of acquired monocular blindness in the world.Around half million people are blind as a result of ocular injuries. Cataract formation is a serious visually challenging sequel after trauma.

Aim:

1. To evaluate visual outcomes following cataract surgeries in the eyes with traumatic cataract.

2. To study the associated ocular morbidities of traumatic cataract.

Materials And Methods: A total number of 50 patients were selected from the outdoor as well indoor patients after fulfilling the inclusion and exclusion criteria. Detailed history, ocular examinations and investigations were performed. Subsequently appropriate surgical management was done and the visual outcome was evaluated on follow ups.

Results: Out of 50 patients 29 (58\%) suffered from blunt trauma and the rest 21( 42\%) from penetrating. Cataract was seen in 39 cases(78\%). SICS with PCIOL implantation was done in 38(78\%)patients and only SICS was done in 5 cases. Lens aspiration with PCIOL. Phaco aspiration with PCIOL were done in 3 and 2 cases each. A vision of 6/6 to 6/18 was achieved in 38(80.85\%) and less than 6/18 in $9(19.15 \%)$ cases.

Associated morbidities found were corneal opacities in $19(38 \%)$, anterior capsule tear with lens matter in A.C in $11(22 \%)$ along with lens subluxation and dislocation in $7(14 \%)$ cases.

CONCLUSION: Early diagnosis followed by proper management definitely plays an important role in better visual outcome after surgery.
\end{abstract}

Keynote: Trauma, cataract, lens dislocation, PCIOL

\section{Introduction}

Ocular trauma is an important, preventable public health problem worldwide. It is the single most important cause of acquired monocular blindness in the world. As many as half a million people in the world are blind as a result of ocular injuries. $40 \%$ of monocular blindness may be related to ocular trauma ${ }^{[1,2)]}$ Cataract formation is a serious visually challenging squeal after trauma. A traumatic cataract may develop after various types of ocular insult, including blunt or perforating trauma as well as ionising, infrared, or ultraviolet radiation Development of cataract during the early years of life leads not only to visual impairment due to the cataract itself, but also to amblyopia. Any significant stimulus deprivation during the amblyogenic age can profoundly affect the further visual development.The damage to other ocular tissues due to trauma may compromise visual gains in eyes operated for traumatic cataract. Both penetrating and concussion injuries can cause cataract. The type of trauma, extent of lenticular involvement and associated damage to the ocular structures go a long way in determining the ultimate prognosis.Surgery for traumatic cataract can be primary or secondary. The surgical approach, potential use of capsular tension devices and choice of IOL(Intra Ocular Lens) are all dictated by the inherent integrity of the zonules, lens capsule, and other associated anterior segment structures. Excluding other intrinsic causes of visual dysfunction, an eye with a traumatic cataract is amenable to treatment and has an excellent potential for significant post operative visual improvement A total number of 50 patients with traumatic cataract were included in the study with the aims and objectives

1. To evaluate visual outcome following cataract surgeries in eyes with traumatic cataract

2. To study the associated ocular morbidities of traumatic cataract.

\section{Materials And Methods}

The present study was conducted in Regional Institute Of Ophthalmology, Gauhati Medical College and hospital, Guwahati for a period of one year from june 2013 to may 2014. A total number of 50 patients were selected from the outdoor as well indoor patients. Informed and written consent was obtained from the patients after explaining the purpose of study and the study design 


\section{Inclusion criteria}

Both male and female patients in the age group of 6-55 years who are diagnosed as having unilateral traumatic cataract by history, clinical findings and investigation.

\section{Exclusion criteria}

Patients with central corneal scar, retinal detachment, vitreous heamorrhage, optic nerve avulsion, poor vision before suffering from ocular trauma based on history and earlier medical records and ocular deviation.

\section{Methodology}

A detailed history was taken and thorough ocular examinations including visual acuity, torch light examination, extraocular motility, cover test and cover-uncover test, slit lamp examination, fundoscopy, slit lamp biomicroscopy, extraocular motility, tonometry were done.Keratometry and A-scan biometry for intraocular lens power calculation was done and syringing for lacrimal system patency and B-scan ultrasonography was done in all cases of traumatic cataract A thorough general examination was carried out and pre operative investigations like routine blood analysis, random blood sugar, serum creatinine, $\mathrm{x}$ ray chest, electrocardiogram and routine urine analysis were carried out.All the patients were prepared pre operatively with topical antibiotic and anti inflammatory drugs with systemic antibiotics in all cases 2 days before surgery.Manual SICS(small incision cataract surgery) with PCIOL(posterior chamber intra ocular lens) implantation was done in 38 cases. Only SICS was done in 5 cases due to vitreous loss. Lens aspiration with PCIOL, Phaco aspiration with PCIOL was done in 3 and 2 cases each.Post operative checkup was carried out at 1 week, 2 weeks, and 6 weeks. Visual acquity was recorded at each visit and Final best corrected visual acuity was recorded at 6 weeks.

\section{Results And Observations}

A total number of 50 eyes of 50 patients of traumatic cataract were selected from the outpatients as well as indoor patients. The age range was from 6 years to 55 years (mean age $32.18+/-19.54$ ) years. Out of 50 cases, 33 were males and 17 were females. The male: female ratio was found to be 1.94:1.

Table 1 : age and sex wise distribution of traumatic cataract patients.

\begin{tabular}{|l|l|l|l|}
\hline Age group(years) & Males $(\boldsymbol{\%})$ & Female $(\boldsymbol{\%})$ & Number of cases(\%) \\
\hline$<10$ years & $9(27.27 \%)$ & $4(23.52 \%)$ & $13(26 \%)$ \\
\hline $11-20$ years & $3(9.09 \%)$ & $1(5.88 \%)$ & $4(8 \%)$ \\
\hline $21-30$ years & $2(6.06 \%)$ & $3(17.64 \%)$ & $5(10 \%)$ \\
\hline $31-40$ years & $6(18.18 \%)$ & $2(17.64 \%)$ & $9(18 \%)$ \\
\hline 41-50 years & $5(15.15 \%)$ & $6(23.52 \%)$ & $9(18 \%)$ \\
\hline $51-55$ years & $8(24.24 \%)$ & 0 & $8(16 \% 0$ \\
\hline Total & $33(100 \%)$ & $17(100 \%)$ & $50(100 \%)$ \\
\hline
\end{tabular}

Maximum number of patients was found in less than 10 years of age groups(26\%). A considerable number of patients belong to the working age group i.e. 21-50 years.

Table 2 : Type of trauma causing traumatic cataract $(\mathrm{n}=50)$

\begin{tabular}{|l|l|l|l|}
\hline Type of trauma & Male (\%) & Female (\%) & Number of cases (\%) \\
\hline Blunt & $18(54.55 \%)$ & $11(64.71 \%)$ & $29(58 \%)$ \\
\hline Penetrating & $15(45.45 \%)$ & $6(35.29 \%)$ & $21(42 \%)$ \\
\hline Total & $33(100 \%)$ & $17(100 \%)$ & $50(100 \%)$ \\
\hline df $=1 \mathrm{x}^{\mathbf{2}}=0.9974 \mathrm{p}>0.05$
\end{tabular}

Type of cataract: On analyzing the type of cataract, it was found that 39 cases had total lens opacity, 6 cases had a soft cataract while 5 cases had a rosette cataract.

Table 3: Morphology of traumatic cataract according to the type of injury $(\mathrm{n}=50)$

\begin{tabular}{|l|l|l|l|l|}
\hline Type of cataract & Total cataract & Soft cataract & Rosette cataract & Total \\
\hline Blunt & $23(58.97 \%)$ & 0 & $5(100 \%)$ & $28 \%$ \\
\hline Penetrating & $16(41.03 \%)$ & $6(100 \%)$ & 0 & $22 \%$ \\
\hline Total & $39(100 \%)$ & $6(100 \%)$ & $5(100 \%)$ & $50(100 \%)$ \\
\hline
\end{tabular}

$\mathrm{Df}=2 \mathrm{x}=7.4286 \mathrm{p}<0.01$

Out of the 39 total cataract cases, 23(58.97) suffered from blunt trauma and all the 6(100\%) of soft cataract cases were due to penetrating trauma. Also all the 5 cases of rosette cataract were due to blunt trauma. 
A statistically significant association $(\mathrm{p}<0.01)$ was found between the type of trauma and the morphology of traumatic cataract.

\section{Pre operative visual acuity}

Pre operative visual acuity was recorded in all cases and was found as an important factor affecting the final visual outcome.

Table 4: pre operative visual acuity $(n=50)$

\begin{tabular}{|l|l|l|l|}
\hline Visual acuity & Blunt trauma & Penetrating trauma & Total number of patients(\%) \\
\hline PL/PR & $7(24.13 \%)$ & $10(47.62 \%)$ & $17(34 \%)$ \\
\hline HMCF & $19(65.52 \%)$ & $9(42.56 \%)$ & $28(56 \%)$ \\
\hline CF & $2(6.90 \%)$ & 0 & $2(4 \%)$ \\
\hline CAN & $1(3.45 \%)$ & $2(9.52 \%)$ & $3(6 \%)$ \\
\hline Total & $29(100 \%)$ & $21(100 \%)$ & $50(100 \%)$ \\
\hline
\end{tabular}

$\mathrm{df}=3 \mathrm{x}^{2}=5.2897 \mathrm{p}>0.05$

PL-Perception of light

PR- Projectionof rays

CF- Counting fingers

CAN- Could not be assessed

Our study showed that out of total 29 blunt trauma cases, $19(65.52 \%)$ had a pre-operative visual acuity of HMCF. Out of 21 penetrating trauma cases, 10(47.62\%) cases had a pre-operative visual acuity of PL/PR. The association between type of trauma and pre-operative visual acuity was not found to be statistically significant.Visual acuity of the uninjured eye was recorded in all patients and was found to be within normal limit

\section{Associated ocular morbidities}

Associated ocular morbidities play an important role in determining the ultimate visual prognosis in cases of traumatic cataract.

Table5: Ocular morbidities associated with traumatic cataract

\begin{tabular}{|l|l|l|}
\hline Associated ocular damage & Number of patients & Percentage \% \\
\hline Corneal involvement & 19 & $38 \%$ \\
\hline $\begin{array}{l}\text { Anterior capsular tear with lens matter in } \\
\text { anterior chamber }\end{array}$ & 11 & $22 \%$ \\
\hline Posterior synechia & 8 & $16 \%$ \\
\hline Subluxation/Dislocation of the lens & 7 & $14 \%$ \\
\hline Uveitis & 5 & $10 \%$ \\
\hline Raised Intra ocular pressure & 4 & $8 \%$ \\
\hline Iris injury & 2 & $4 \%$ \\
\hline
\end{tabular}

Out of 50 cases of traumatic cataract, $19(38 \%)$ had corneal involvement in the form of scars, opacity or sealed corneal wound while 11( 22\%) cases had anterior capsular tear with lens matter in AC. Posterior synechiae was found in $8(16 \%)$, subluxation of lens was in 5 while 2 cases had dislocation of lens and uveitis in $5(10 \%)$ cases. Total $4(8 \%)$ patients showed raised IOP. Iris

Types of surgery:

Out of 50 cases of traumatic cataract, 38 underwent SICS(small incision cataract surgery) with posterior chamber intraocular lens implantation. In 5 cases, SICS was done because of posterior capsular rupture with vitreous loss. Secondary IOL implantation was planned in these cases. Manual/Phaco aspiration of the cataractous lens with PCIOL implantation was done in 3 cases. Phacoemulsification with PCIOL implantation was done in 2 cases, Sics with sclera fixated IOL and SICS with anterior vitrectomy was done in 1 case each.

Table6: Types of surgery in traumatic cataract patients $(n=50)$

\begin{tabular}{|l|l|l|}
\hline Type of surgery & Number of patients & Percentage \% \\
\hline SICS with PCIOL & 38 & $76 \%$ \\
\hline SICS & 5 & $10 \%$ \\
\hline Lens aspiration plus PCIOL & 3 & $6 \%$ \\
\hline Phacoemulsification plus PCIOL & 2 & $4 \%$ \\
\hline SICS with Scleral Fixated IOL & 1 & $2 \%$ \\
\hline SICS with anterior vitrectomy & 1 & $2 \%$ \\
\hline Total & 50 & $100 \%$ \\
\hline
\end{tabular}




\section{Intra operative complications}

Complications during surgery either due to trauma or surgery or both was noted as vitreous loss in patients with posterior capsular rupture in 5 cases $(10 \%)$

\section{Post operative complications}

Posterior capsular opacification was a late complication noted in 10(20\%) patients mostly of paediatric age group. These cases were planned for Nd-Yag capsulotomy after 6 weeks post operative. Striate keratopathy was noted in immediate post operative period in 2(4\%) patients. Hyphaema, acquired iris coloboma and IOL decentration was noted in 1 case $(2 \%)$ each.

Table 7: Post-operative complications in patients of traumatic cataract

\begin{tabular}{|l|l|l|}
\hline Postoperative complication & No. of eyes & Percentage $\%$ \\
\hline Posterior capsular opacification & 10 & $20 \%$ \\
\hline Striate keratopathy & 2 & $4 \%$ \\
\hline Hyphaema & 1 & $2 \%$ \\
\hline Acquired iris coloboma & 1 & $2 \%$ \\
\hline IOL decentration & 1 & $2 \%$ \\
\hline
\end{tabular}

\section{Visual results}

The final best corrected visual acuity ( BCVA) was measured 6 weeks after surgery. 3 cases lost follow up out of which one was 6years, one was47years and one was 53years old.

Table8: Final BCVA at 6 weeks post operative period

\begin{tabular}{|l|l|l|}
\hline Final BCVA & Number of eyes & Percentage(\%) \\
\hline $6 / 6$ & 4 & $8.51 \%$ \\
\hline $6 / 9$ & 8 & $17.02 \%$ \\
\hline $6 / 12$ & 16 & $34.04 \%$ \\
\hline $6 / 18$ & 10 & $21.27 \%$ \\
\hline $6 / 24$ & 4 & $8.51 \%$ \\
\hline $6 / 36$ & 2 & $4.25 \%$ \\
\hline $6 / 60$ & 3 & $6.38 \%$ \\
\hline$<6 / 60$ & 0 & 0 \\
\hline Total & 47 & $100 \%$ \\
\hline
\end{tabular}

The final visual acuity ( best corrected visual acuity ) was assessed at the end of 6 weeks . None of the patient had visual acuity less than $6 / 60$.

Table 9: BCVA in relation to duration between trauma and surgery.

\begin{tabular}{|l|l|l|l|}
\hline $\begin{array}{l}\text { Duration between trauma and } \\
\text { surgery }\end{array}$ & $6 / 6-6 / 18$ & $<6 / 18-3 / 60$ & Total \\
\hline$<1$ month & $8(21.05 \%)$ & $1(11.11 \%)$ & $9(19.15 \%)$ \\
\hline 1 month - 1 year & $29(76.32 \%)$ & $7(77.78 \%)$ & $36(74.48 \%)$ \\
\hline$>1$ year & $1(2.63 \%)$ & $1(11.11 \%)$ & $2(4.27 \%)$ \\
\hline Total & $38(100 \%)$ & $9(100 \%)$ & $47(100 \%)$ \\
\hline
\end{tabular}

$\mathrm{df}=2 \mathrm{x}^{2}=1.6237 \mathrm{p}>0.05$

Out of the 38 patients with BCVA of $6 / 6-6 / 18,29(76.32 \%)$ the duration between trauma and surgery was 1 month to 1 year. Out of 9 patients with BCVA of $<6 / 18$ to $3 / 60$, in 7 cases $(74.48 \%)$, the duration between trauma and surgery was 1 month to one year. No statistically significant association was noticed between final $\mathrm{BCVA}$ and the duration between trauma and surgery.

Table 10: Pre-operative and post-operative Final BCVA in traumatic cataract patients $(\mathrm{n}=47)$.

\begin{tabular}{|l|l|l|l|l|l|}
\hline $\begin{array}{l}\text { Post- } \\
\text { operative } \\
\text { final BCVA }\end{array}$ & PL/PR & HMCF & CF & CNA & \\
\hline $6 / 6-6 / 18$ & $11(73.33 \%)$ & $24(85.71 \%)$ & $2(100 \%)$ & $1(59 \%)$ & $38(80.85 \%)$ \\
\hline$<6 / 18-3 / 60$ & $4(26.67 \%)$ & $4(14.29 \%)$ & 0 & $1(50 \%)$ & $9(19.15 \%)$ \\
\hline Total & $15(100 \%)$ & $28(! 00 \%)$ & $2(100 \%)$ & $2(100 \%)$ & $47(100 \%)$ \\
\hline
\end{tabular}

Out of tatal 47 patients, the post-operative final BCVA was found to be $6 / 6$ to $6 / 18$ in 38 (80.85\%) patients, irrespective of their pre-operative visual acuity. 


\section{Discussion}

Age:

Age wise analysis showed that majority of the cases occurred in $<10$ years of age group. This is most probably because children are more active ,meet with accidents more often while playing, and lack of adult supervision is also responsible for trauma. Daljit Singh et al ${ }^{[5]}$ also showed similar age group distribution.

Sex:

This study showed a male preponderance (66\%) .Men seem to be more exposed to ocular trauma because they spend most of their time outdoors in farming or industrial related activities. Bhatia IM ,Panda et $\mathrm{al}^{[6]}$ and Murli Krishnamachiary ${ }^{[7]}$ et al found a similar male preponderance in their study populations.

Type of trauma:

In this study blunt trauma was common mode of injury i.e. in $29(58 \%)$ cases. Penetrating trauma was seen in $21(42 \%)$ cases . Similar findings were reported by Gagandeep Brar ${ }^{[8]}$ and Marcus Blum ${ }^{[9]}$.

\section{Morphology of traumatic cataract:}

Most patients , 39(78\%) had total cataract, among which 6 cases (12\%) had soft cataract, while 5 cases $(10 \%)$ had rosette shaped cataract. A statistically significant association $(p<0.01)$ was found between the type of trauma and the morphology of traumatic cataract. Krishnamachary $\mathrm{M}^{[7]}$ et al observed total cataract in $53.2 \%$ cases. Shah $\mathrm{M}^{[10]}$ et al found white soft cataract in majority of (60\%) patients and total cataract in $26.6 \%$ cases.

\section{Pre-operation BCVA:}

Out of the total 29 blunt trauma cases, 19(65.52\%) had a pre-operative visual acuity of HMCF in the injured eye. Out of the total 21 penetrating trauma cases, 10 had a pre-operative visual acuity of PL/PR. The association between type of trauma and pre-operative visual acuity of the injured eye was not found to be statistically significant $\left(\mathrm{X}^{2}=5.2897, \mathrm{p}>0.05\right)$.

Visual acuity ranged from $20 / 200$ or worse in $97.7 \%$ of patients preoperatively in the study done by Krisnamachary and Varsha $\mathrm{R}^{(7)}$ in 1997. Also according to YING-Nan Xu, Yu-Sen Huang ${ }^{[11]}$ et al (2013) in their study of 117 traumatic cataract patients, there were no significant difference in the pre-operation BCVA between open-globe and closed-globe injuries $\left(\mathrm{X}^{2}=3.889, \mathrm{p}=0.274\right)$.

\section{Associations of traumatic cataract :}

Out of 50 cases, $19(38 \%)$ presented with corneal involvement in the form of scars, opacity or sealed corneal wound.Posterior synechiae was noted in $16 \%$ patients. Uveitis was observed in $10 \%$ cases. Raised IOP was noted in $4(8 \%)$ patients .Iris injury in the form of iridodialysis was found in 1 patient and traumatic mydriasis in 1 patient. $22 \%$ suffered from anterior capsular tear with lens matter in AC .Subluxation of lens was noted in 5 cases, while lens dislocation was noted in 2 cases. Similar pattern of distribution of other associated ocular morbidities has been observed by Marcus Blum ${ }^{[9]}$ et al, Krishnamachiary ${ }^{[7]}$ et al and Daljit Singh ${ }^{[5]}$ et al.

\section{Type of surgery:}

In this study out of 50 cases, 38 underwent SICS (small incision cataract surgery) with posterior chamber intraocular lens implantation, in 5 cases SICS was done, manual/ Phaco Lens aspiration with PCIOL was done in 3 cases whereas Phacoemulsification with PCIOL implantation was done in 2 cases. SICS with Scleral Fixated IOL and SICS with Anterior Vitractomy was done each in 1 case. Krishnamachary M .Rathi V ${ }^{[7]}$ et al in 1998 reviewed 237 children who developed traumatic cataracts.Extra capsular cataract extraction with IOL Implantation Was performed in $65.67 \%$ of patient. Chuang LH,Lai CC ${ }^{[12]}$ in 2005 evaluated 30 pts with traumatic cataract in open globe injury.All patients underwent cataract extraction with primary repair of the penetrating wound ,18 eyes $(60 \%)$ underwent pars plana vitrectomy with lensectomy and 12 eyes $(40 \%)$ underwent lens aspiration or extracapsular cataract extraction.

\section{Intraoperative complications-}

Complications during surgery can be either due to trauma or surgery or both.Vitreous loss was observed in $5(10 \%)$ patients with posterior capsular rupture. Shoeb Ahmed ${ }^{[13]}$ reported $10(20 \%)$ cases of vitreous loss in his study of 50 traumatic cataract cases in 1998.

\section{Post-operative complications -}

Posterior capsule opacification was a late complication noted in $10(20 \%)$ patients mostly of pediatric age group. Striate keratopathy was observed in immediate post operative period in $2(4 \%)$ patients. Hyphema, 
acquired iris coloboma and IOL decentration was noted in one case each . Murali Krishnamachary,Varsha Rathi ${ }^{[7]}$ noted PCO in $42.9 \%$ patients. Renuka Srinivasan ${ }^{[14]}$ et al reported Striate keratopathy in $8.8 \%$, Hyphaema in $11.8 \%, \mathrm{PCO}$ in $9 \%$ and IOL decentration in $8.2 \%$ cases.

\section{Visual Result :}

Final BCVA was documented in 47 cases as 3 cases were lost to follow up. The final visual acuity was graded into three categories based on 'WHO Vision Categories'- vision of $<3 / 60$ as blind, Vision of 3/60 to $<6 / 18$ as visually impaired (low vision ) and $6 / 18$ and above as adequate vision. At the end of one and half months final BCVA was better than $6 / 18$ in $38(80.85 \%)$ cases and $<6 / 18-3 / 60$ in $9(19.15 \%)$ cases .None of the patient had visual acuity less than $6 / 60$

Daljit Singh ${ }^{[5]}$ et al studied 61 cases of traumatic cataract and noted final visual acuity of 6/6 to 6/12 in $79 \%$ of cases after surgery. Murali Krishnamachary ${ }^{[7]}$ noted postoperative visual acuity of $20 / 60$ or better in $74.1 \%$ patients after ECCE with IOL Implantation. Synder A ,Kobielska ${ }^{[15]}$ noted that good visual acuity i.e $6 / 6$ to $6 / 18$ was achieved in $71.45 \%$ cases after undergoing Extracapsular cataract extraction in traumatic cases.

\section{Conclusion}

Early diagnosis followed by proper management definitely plays an important role in better visual outcome after surgery in cases of traumatic cataract. Stress should therefore be put on ocular safety measures and awareness of the society against ocular injuries occurring at work place and home because prevention is better than cure. Also the importance of early reporting and adequate follow up especially in children suffering from traumatic cataract needs to be emphasized to the masses.

\section{References}

[1]. Thylefors B. Epidemiological patterns of ocular trauma. Australian and New Zealand journal of ophthalmology. 1992 May 1;20(2):95-8.

[2]. Négrel AD, Massembo-Yako B, Botaka E, Minassian DC, Coddy-Zitsamélé R. [Prevalence and causes of blindness in the Congo]. Bull World Health Organ. 1990;68(2):237-43

[3]. Johns KJ, Feder RS, Bowes Hamill M. Lens and cataract. AAO Basic and Clinical Science Course Series. San Francisco: The Foundation for the American Academy of Ophthalmology. 2001:50-4.

[4]. Zaman M, Sofia I, Muhammad DK. Frequency and visual outcome of traumatic cataract. J Postgrad Med Inst 2006; 20:330-4.

[5]. Singh D et al. The role of intraocular lens in traumatic cataract. Indian J Ophthalmol, 31; 294-297.

[6]. Bhatia IM, Panda A, Sood NN. Management of traumatic cataract. 1983; 31:290-293.

[7]. Krishnamachari M, Rati V, Gupta J. Management of traumatic cataract in children. Jr. of Cat \& Ref surgery, $1997 ; 23(1)$ : 681-7.

[8]. Brar GS, Ram J, Pandav SS, Reddy SS. Postoperative complications and visual results in uniocular pediatric traumatic cataract. Ophthalmic Surg Lasers, 2001: 32:233-8.

[9]. Blum M, Tetz MR. Treatment of traumatic cataract. J. Cataract Refract Surg, April 1996.

[10]. Shah M, Shah S, Shah S, et al. Visual recovery and predictors of visual prognosis after managing traumatic cataracts in 555 patients. Indian J Ophthalmol.2011; 59 (3): 217-222.

[11]. Xu YN, Huang YS, Xie LX. Pediatric traumatic cataract and surgery outcomes in eastern China: a hospital-based study. International journal of ophthalmology. 2013;6(2):160-164.

[12]. Chuang LH, Lai CC. Secondary intraocular lens implantation of traumatic cataract in open-globe injury. Canadian Journal of Ophthalmology/Journal Canadien d'Ophtalmologie. 2005 Aug 1;40(4):454-9.

[13]. Ahmed SS. Traumatic cataract-Newer Perspectives. Jr. of TNOA. 1998 XXXIII Jun;38(2):31-3.

[14]. Srinivasan R Kumadhan et al. Traumatic cataract-factors affecting visual outcome. Jr. of TNOA, XXXVIII(1): 45-48.

[15]. Synder A, Kobielska D, Omulecki W. Intraocular lens implantation in traumatic cataract. Klinika oczna. 1998 Dec;101(5):343-6. 